\title{
Not Applicable
}

National Cancer Institute

\section{Source}

National Cancer Institute. Not Applicable. NCI Thesaurus. Code C48660.

Determination of a value is not relevant in the current context. 